\title{
Epidemiología de la infección asociada a catéter venoso central
}

\author{
ÁNGELA LILIANA LONDOÑO F. ${ }^{1}$ MARGARITA ARDILA F. ${ }^{2}$, DAVID OSSA P. ${ }^{3}$. \\ 1. MD especialista en Epidemiología, PhD Medicina Preventiva y Salud Pública. Facultad de Ciencias de la Salud, \\ Universidad del Quindío. \\ 2. Licenciada en Enfermería Superior. Especialista en Gerencia en Servicios de Salud. Facultad de Ciencias de la Salud, \\ Universidad del Quindío. \\ 3. MD Especialista en Pediatría. Facultad de Ciencias de la Salud, Universidad del Quindío.
}

\begin{abstract}
Epidemiology of Infections of Central Venous Catheters

Objetive: To identify the frequency of infections related to Central Cenous Catheters (CVC)in children and determine if the colonization of the line predicts infection. Patients and Methods. A longitudinal descriptive study of 109 CVC. Colonization was detected at the entry point and connection points of the lines with a semi quantitative method. Definitions were made according to CDC (Atlanta) Criteria. Results: The incidence of infection into the blood stream was $11 \%$, density of incidence $9 / 1000$ days/ catheter. Risk factors were identified: a) surgery (RR 4,2 IC95\% 1,5-11,7), b) ostomies (RR 4,0 IC95\% 1,4-11,4), and c) colonization in line connections (RR 3,9 IC95\% 1,2-12,3). Agents identified were Staphylococcus coagulasa (-) 83,4\% and Candida albicans $16,6 \%$. Incidence of local infection was 5,5\%. Sensitivity, specificity and (+) predictive value of cultures of cultures and connections were very low. Conclusions: In this experience, the incidence of infection associated to peripheral lines was quite high. Colonization of insertion and connections does not predict infection. The implemmentation of Bundle is recommended to prevent these infections.
\end{abstract}

(Key words: Bacteremia, infections related to catheters, intrahospital infections, infection control).

Rev Chil Pediatr 2011; 82 (6): 493-501

\section{RESUMEN}

Objetivo: Identificar frecuencia de infecciones relacionadas con catéter venoso central en niños y determinar si la colonización del catéter predice la infección. Pacientes y Métodos: Se hizo un estudio descriptivo longitudinal en 109 catéteres venosos centrales; se detectó colonización en sitio de entrada y conexiones de los catéteres con método semicuantitativo. Las definiciones se realizaron con los criterios de los Centros de Control de Enfermedades de Atlanta. Resultados: La Incidencia de infección del torrente sanguíneo fue 11\%, la densidad de incidencia 9/1000 días/ catéter; se identificaron como factores de riesgo la cirugía (RR 4,2 IC95\% 1,5-11,7), las ostomías (RR 4,0 IC95\% 1,4-11,4) y la colonización en conexiones del catéter (RR 3,9

Trabajo recibido el 31 de mayo de 2011, devuelto para corregir el 31 de agosto de 2011, segunda versión el 26 de septiembre de 2011, aceptado para publicación el 14 de octubre de 2011.

Investigación financiada por la Universidad del Quindío, Armenia-Quindío-Colombia.

Correspondencia a:

Liliana Londoño F.

E-mail: angelalilianal@uniquindio.edu.co 
IC95\% 1,2-12,3); fueron provocadas por Staphylococcus coagulasa (-) 83,4\% y Candida albicans 16,6\%. La incidencia de infección local fue de 5,5\%. La sensibilidad, especificidad y valor predictivo $(+)$ de los cultivos en inserción y conexiones fueron muy bajos. Conclusiones: En esta experiencia se encontró una elevada incidencia de infección asociada a catéteres de inserción periférica; la colonización de inserción y conexiones no fueron predictores de la infección; se recomienda implementar Bundle para prevención de estas infecciones.

(Palabras clave: Bacteriemia, infecciones relacionadas con catéteres, infección hospitalaria, control de infecciones).

Rev Chil Pediatr 2011; 82 (6): 493-501

\section{Introducción}

Los catéteres venosos centrales (CVCs) son indispensables en la práctica médica hoy en día como paso esencial para la utilización de gran variedad de técnicas de monitorización y tratamiento, facilitan el cuidado de los niños con enfermedades crónicas o graves; sin embargo, su uso se asocia con frecuencia con complicaciones infecciosas locales o sistémicas entre las cuales la infección del torrente sanguíneo es la más frecuente con consecuencias como hospitalización prolongada e incremento en morbilidad, mortalidad y $\operatorname{costos}^{1-4}$. La mayoría de las infecciones asociadas con la atención sanitaria (IAAS) están asociadas a dispositivos médicos y la infección del torrente es una de las principales IAAS 5 .

La patogénesis de las infecciones asociadas a CVCs es multifactorial y compleja, resulta de la migración de organismos superficiales relacionados con la piel del sitio de inserción que migran por el tracto cutáneo del catéter con colonización eventual de la punta, este mecanismo extraluminal lleva a bacteriemia en los primeros días de cateterización ${ }^{2-4}$. La ruta intraluminal donde la contaminación ocurre por una inadecuada limpieza de las conexiones durante la conexión y desconexión de los sistemas sobre todo en catéteres de varios lúmenes y múltiples vías, es la vía más frecuente de infección después de la primera semana de cateterización. Aunque menos común, puede ocurrir siembra hematógena de un foco distante de infección o la contaminación intrínseca del líquido infundido ${ }^{1-4}$. Intervienen también el material del cual está hecho el catéter siendo menor la frecuencia con CVCs de poliuretano que de silicona; la reacción de "cuerpo extraño" que induce alteraciones locales en los tejidos circundantes ${ }^{4,6}$ y las propiedades de adhesión de los microorganismos que forman biofilms bacterianos altamente resistentes a muchos agentes antimicrobianos ${ }^{4,7,8}$. Otros factores de riesgo se relacionan con el paciente (edades extremas, inmunosupresión), con el CVC (número de lúmenes, localización, duración, inserción y cuidados) y con infusión de nutrición parenteral lipídica ${ }^{1,2,4}$.

\section{Objetivos}

Identificar la frecuencia de las infecciones relacionadas con $\mathrm{CVC}$, describir la relación con algunos factores de riesgo y calcular el valor diagnóstico de la colonización de la piel en el sitio de inserción y las conexiones para la infección del torrente sanguíneo.

\section{Pacientes y Método}

Se realizó un estudio descriptivo longitudinal prospectivo, en 109 CVCs instalados en 82 pacientes pediátricos de las unidades de recién nacidos y pediatría de una institución de tercer nivel en Armenia-Colombia, durante 8 meses entre 2009 y 2010. Se incluyeron: CVCs de inserción periférica (PICC) y centrales no tunelizados (CNT) instalados en la institución, duración mínima en el sitio 48 horas; se excluyeron CVCs en pacientes que salían de la institución y luego reingresaran con el catéter. Las definiciones de Infección del torrente sanguíneo asociada a vía central y confirmada por laboratorio (ITS), infección local y colo- 
INFECCIÓN Y CATÉTER VENOSO CENTRAL

nización se realizaron con los criterios de los CDC de Atlanta ${ }^{1,5}$. Se incluyeron variables independientes de características de los catéteres (sitio de inserción, lúmenes, instalación y motivo de retirada; con cuidados y mantenimiento del CVC (antiséptico para limpieza, cambio de sistemas de infusión para administrar soluciones) y usos (transfusiones, nutrición parenteral, fluidoterapia y medicamentos).

\section{Técnicas y procedimientos}

En el Centro de investigaciones Biomédicas de la Universidad del Quindío se procesaron segmentos de catéter, muestras semanales de piel del sitio de inserción y conexiones de los CVCs y hemocultivos. Las muestras fueron tomadas con técnica estéril por una enfermera; se cortaron asépticamente 3-4 cm del extremo distal del catéter en aquellos niños con sospecha de infección. Se utilizó el método semicuantitativo para identificar colonización según la técnica descrita por Maki en 1977, rodando varias veces el segmento de catéter o el hisopo sobre la superficie de una placa de agar sangre con pinzas estériles; se consideró colonizado $\geq 15$ ufc por placa ${ }^{9,10}$. A las muestras se les realizó cultivo, coloración de Gram e identificación de especie y pruebas de susceptibilidad con el método de sensibilidad por difusión en disco según las recomendaciones del NCCLS (Comité Nacional para Estándares de Laboratorios Clínicos).

\section{Protocolo hospitalario}

Todos los CVCs a utilizar fueron de poliuretano, se hizo capacitación sobre las normas recomendadas por los $\mathrm{CDC}^{1}$. Se cumplieron los aspectos bioéticos según lo establecido por el Ministerio de Salud de Colombia en la Resolución 8430 de 1993 que define las normas técnicas y científicas para investigación en salud.

Se procesó la información en SPSS versión 14, se calcularon: incidencia acumulada (IA \%) y densidad de incidencia por 1000 días/catéter (DI). Se hizo distribución de frecuencias del perfil de resistencia. Se exploró relación de variables con ITS, para variables cualitativas con la distribución de $\chi^{2}$ y a las cuantitativas se aplicó la prueba de Kolgomorov Smirnov para definir pruebas paramétricas. Se calculó valor predictivo de la colonización en el punto de entrada y conexión para el diagnóstico de ITS.

\section{Resultados}

\section{Características y cuidados de los CVCs}

$72,4 \%$ fueron instalados en pabellón, $25,5 \%$ en la unidad de recién nacidos y $2,0 \%$ en cuidados intensivos. Un $22,9 \%$ (n:25) fueron PICC y 77,1\% (n: 84) CNT. Los CNT se observaron en femoral $66,3 \%$, subclavia $8,9 \%$ y yugular $5,0 \%$; un $96,2 \%$ fueron unilúmen. Se utilizaron para nutrición parenteral $50 \%$, transfusión de hemoderivados 19,3\% y el resto para fluidoterapia y medicamentos. El cambio de los sistemas de infusión se realizó en promedio cada 4,3 días (IC95\% 3,8-4,7), el 18,2\% se cambiaron en menos de 48 horas, 63,0\% 48-72 horas y $18,2 \%$ cada 96 y más horas. El antiséptico utilizado para la limpieza del sitio de inserción fue la povidona yodada en $40,4 \%$, povidona yodada o alcohol o clorhexidina $46,1 \%$ y alcohol o clorhexidina $13,5 \%$.

El promedio de duración de los CVCs fue de 13,1 días (IC95\% 11,4-14,8), los PICC 10,2 días (IC95\% 6,8-13,7) y los CNT 13,9 días (IC95\% 11,9-15,8). Fueron retirados en un $74,5 \%$ por finalización de la indicación; $9,8 \%$ problemas mecánicos (acodamiento, obstrucción, infiltración), 5,9\% retiro accidental; $4,9 \%$ infección local, $1 \%$ sospecha de infección del torrente sanguíneo (IT) y 3,9\% cambio con guía.

\section{Infección asociada a CVC y factores relacionados}

Se presentó infección local (IL) en el sitio de entrada de 6 CVCs (4 instalados en femoral, 1 en yugular y 1 en basílica). Infección del torrente sanguíneo confirmada por laboratorio (ITS) se asoció a 12/109 CVCs (figura 1). Las ITS fueron provocadas por Staphylococcus coagulasa (-) $(83,4 \%)$; y por Candida albicans $(16,6 \%)$.

\section{Frecuencia de colonización y etiología}

Se cultivaron 19,3\% (21/109) de los CVCs retirados y en 52,4\% (11 segmentos) se aislaron Staphylococcus coagulasa, hongos del gé- 

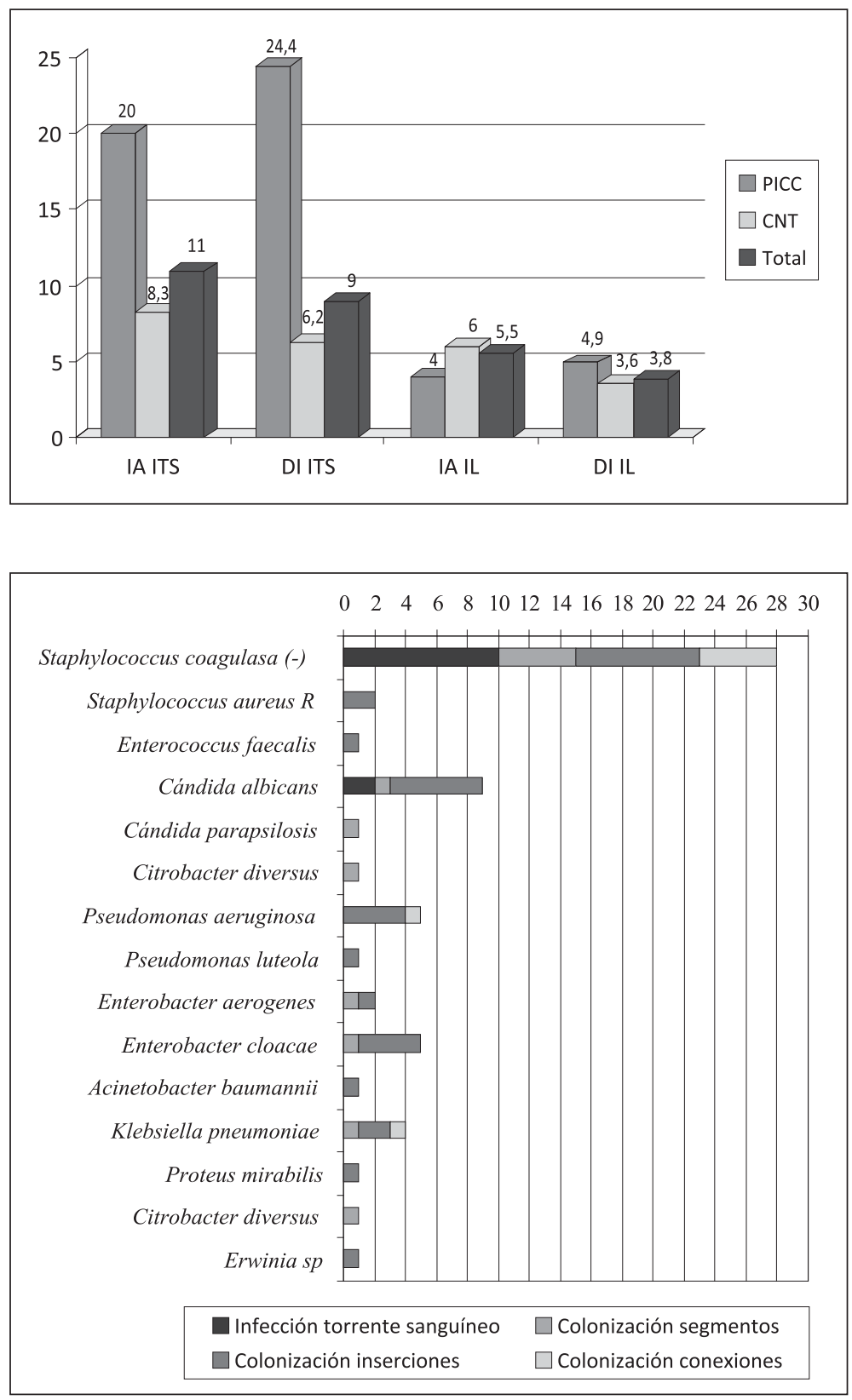

Figura 1. Incidencia de Infección del torrente sanguíneo (ITS) y de infección local (IL). IA: Incidencia acumulada \%; DI: Densidad de incidencia por 1000 días/catéter; PICC: CVC inserción periférica; CNT: CVC de inserción central no tunelizado. nero Cándida y Enterobacteriaceae. En 34\% de los cultivos realizados en el sitio de inserción se aislaron Staphylococcus coagulasa (-), Staphylococcus aureus, Enterococcus faecalis, Cándida albicans, Enterobacteriaceae y bacilos Gram negativos no fermentadores " $B N F$ ". En 7,4\% de las muestras tomadas en las conexiones se aislaron Staphylococcus coagulasa (-) y Gram negativos (figura 2).

\section{Perfil de sensibilidad y resistencia a los antimicrobianos}

Se realizó perfil de sensibilidad y resistencia para todos los cultivos (colonizaciones e ITS). Sólo un 17,9\% de los aislamientos de Staphylococcus coagulasa (-) fueron sensibles a todos los antibióticos; Staphylococcus aureus mostró resistencia. De los Gram negativos aislados fueron sensibles a todos los anti- 
bióticos: Pseudomonas luteola, Acinetobacter baumannii y Erwinia sp; los demás mostraron resistencia a uno o más antibióticos (tablas 1A y 1B).

\section{Variables relacionadas con la ITS}

Se identificaron factores relacionados significativamente con la ITS: procedimientos quirúrgicos o alguna ostomía y la colonización de las conexiones de los CVCs. Se encontraron diferencias en la incidencia pero no estadísti- camente significativas en presencia de: ventilación mecánica, nutrición parenteral, transfusión a través del CVC, infección local, y colonización en sitio de entrada. No se observaron diferencias con las demás variables analizadas (tabla 2).

\section{Variables relacionadas con la colonización de sitio de inserción y conexiones}

Se colonizaron un $40 \%$ de los CVCs con sitio de entrada en yugular; 33,3\% subclavios,

Tabla 1A. Gram positivos. Proporción de resistencia y sensibilidad a antibióticos (n y \%)

\begin{tabular}{|c|c|c|c|c|c|c|c|c|c|c|c|c|}
\hline & AMP & OXA & SAM & CEP & CFZ & IPM & CIP & LVX & GEN & CLI & $\mathbf{S}$ & $\mathbf{n}$ \\
\hline $\begin{array}{l}\text { Staphylococcus } \\
\text { coagulasa (-) }\end{array}$ & $\begin{array}{c}7 \\
(25,0)\end{array}$ & $\begin{array}{c}20 \\
(71,4), \\
\text { I: } 1 \\
(3,6)\end{array}$ & $\begin{array}{c}7 \\
(25,0)\end{array}$ & $\begin{array}{c}1 \\
(3,6)\end{array}$ & $\begin{array}{c}7 \\
(25,0)\end{array}$ & $\begin{array}{c}7 \\
(25,0)\end{array}$ & $\begin{array}{c}6 \\
(21,4)\end{array}$ & $2(7,1)$ & $\begin{array}{c}18 \\
(64,3)\end{array}$ & $\begin{array}{c}15 \\
(53,6)\end{array}$ & $\begin{array}{c}5 \\
(17,9)\end{array}$ & 28 \\
\hline $\begin{array}{l}\text { Staphylococcus } \\
\text { aureus }\end{array}$ & & $\begin{array}{c}2 \\
(100)\end{array}$ & $\begin{array}{c}1 \\
(50,0)\end{array}$ & & & & & & $\begin{array}{c}2 \\
(100)\end{array}$ & $\begin{array}{c}2 \\
(100)\end{array}$ & 0 & 2 \\
\hline $\begin{array}{l}\text { Enterococcus } \\
\text { faecalis }\end{array}$ & & & & & & & & & & & $\begin{array}{c}1 \\
(100)\end{array}$ & 1 \\
\hline
\end{tabular}

Tabla 1B. Gram negativos. Proporción de resistencia y sensibilidad a antibióticos (n y \%)

\begin{tabular}{|c|c|c|c|c|c|c|c|c|c|c|c|c|c|c|c|c|c|}
\hline & AMP & PIP & SAM & TZP & TCC & CEP & CFZ & CXM & CFP & FEP & CSL & MEM & ATM & GEN & AMK & s & $n$ \\
\hline $\begin{array}{l}\text { Citrobacter } \\
\text { diversus }\end{array}$ & & & & & & & & & $\begin{array}{c}1 \\
(100)\end{array}$ & & & & & $\begin{array}{c}1 \\
(100)\end{array}$ & $\begin{array}{c}1 \\
(100)\end{array}$ & 0 & 1 \\
\hline $\begin{array}{l}\text { Pseudomonas } \\
\text { aeruginosa }\end{array}$ & & & & $\begin{array}{l}\text { l:1 } \\
(20)\end{array}$ & & & & & & $\begin{array}{c}1 \\
(20)\end{array}$ & 1 & $\begin{array}{c}2 \\
(40)\end{array}$ & $\begin{array}{c}1 \\
(20)\end{array}$ & & $\begin{array}{l}\text { l:1 } \\
(20)\end{array}$ & $\begin{array}{c}2 \\
(40)\end{array}$ & 5 \\
\hline $\begin{array}{l}\text { Pseudomonas } \\
\text { luteola }\end{array}$ & & & & & & & & & & & & & & & & $\begin{array}{c}1 \\
(100)\end{array}$ & 1 \\
\hline $\begin{array}{l}\text { Enterobacter } \\
\text { aerogenes }\end{array}$ & 1 & & $\begin{array}{c}2 \\
(100)\end{array}$ & & $\begin{array}{c}1 \\
(50)\end{array}$ & $\begin{array}{c}1 \\
(50)\end{array}$ & $\begin{array}{c}1 \\
(50)\end{array}$ & $\begin{array}{c}1 \\
(50)\end{array}$ & & & & & & $\begin{array}{c}1 \\
(50)\end{array}$ & $\begin{array}{c}1 \\
(50)\end{array}$ & 0 & 2 \\
\hline $\begin{array}{l}\text { Enterobacter } \\
\text { cloacae }\end{array}$ & & & $\begin{array}{c}4 \\
(80)\end{array}$ & & & & & & & $\begin{array}{c}1 \\
(20), \\
1: 1 \\
(20)\end{array}$ & & & $\begin{array}{c}1 \\
(20)\end{array}$ & & $\begin{array}{c}1 \\
(20)\end{array}$ & $\begin{array}{c}1 \\
(20)\end{array}$ & 5 \\
\hline $\begin{array}{l}\text { Acinetobacter } \\
\text { baumannii }\end{array}$ & & & & & & & & & & & & & & & & $\begin{array}{c}1 \\
(100)\end{array}$ & 1 \\
\hline $\begin{array}{l}\text { Klebsiella } \\
\text { pneumoniae }\end{array}$ & & & $\begin{array}{c}3 \\
(75)\end{array}$ & & & & & & & $\begin{array}{c}2 \\
(50)\end{array}$ & $\begin{array}{c}1 \\
(25)\end{array}$ & & $\begin{array}{c}3 \\
(75)\end{array}$ & & $\begin{array}{l}\text { l:1 } \\
(25)\end{array}$ & 0 & 4 \\
\hline $\begin{array}{l}\text { Proteus } \\
\text { mirabilis }\end{array}$ & $\begin{array}{c}1 \\
(100)\end{array}$ & $\begin{array}{c}1 \\
(100)\end{array}$ & & & & $\begin{array}{l}\text { l:1 } \\
(100)\end{array}$ & & & & & & & & $\begin{array}{c}1 \\
(100)\end{array}$ & $\begin{array}{c}1 \\
(100)\end{array}$ & 0 & 1 \\
\hline $\begin{array}{l}\text { Citrobacter } \\
\text { diversus }\end{array}$ & & & & & & & & & $\begin{array}{c}1 \\
(100)\end{array}$ & & & & & $\begin{array}{c}1 \\
(100)\end{array}$ & $\begin{array}{c}1 \\
(100)\end{array}$ & 0 & 1 \\
\hline Erwinia sp & & & & & & & & & & & & & & & & $\begin{array}{c}1 \\
(100)\end{array}$ & 1 \\
\hline
\end{tabular}

S: Sensible; n: \# de aislamientos de cada germen; I: resistencia intermedia. Siglas de antibióticos, según WHONET: Ampicilina (AMP), Ampicilinasulbactam (SAM), Aztreonam (ATM), Cefalotina (CEP), Cefazolina (CFZ), Cefepime (FEP), Cefoperazona (CFP), Cefoperazona/ sulbactam (CSL), Cefuroxima (CXM), Ciprofloxacina (CIP), Clindamicina (CLI), Gentamicina (GEN), Imipenem (IPM), , Levofloxacina (LVX), Meropenem (MEM), Oxacilina (OXA) ,Piperacilina (PIP), Piperacilina-tazobactam (TZP), Ticarcilina /ac clavulánico (TCC) 
LONDOÑO A. y cols.

Tabla 2. Relación entre Infección del torrente sanguíneo (ITS) y factores de riesgo

\begin{tabular}{|c|c|c|c|c|c|c|}
\hline \multirow[t]{3}{*}{ Factores } & \multicolumn{4}{|c|}{ ITS n: 12/109 } & \multirow[t]{3}{*}{ RR (IC95\%) } & \multirow[t]{3}{*}{$\chi^{2}(\mathbf{p})$} \\
\hline & \multicolumn{2}{|c|}{ Sí } & \multicolumn{2}{|c|}{ No } & & \\
\hline & $\mathbf{n}$ & $\%$ & $\mathbf{n}$ & $\%$ & & \\
\hline Cirugía & 6 & 28,6 & 6 & 6,8 & $4,2(1,5-11,7)$ & $8,2(0,004)$ \\
\hline Ostomizado & 4 & 33,3 & 8 & 8,2 & $4,0(1,4-11,4)$ & $6,9(0,009)$ \\
\hline Ventilación mecánica & 6 & 16,7 & 6 & 8,2 & $1,1 \quad(1,0-1,3)$ & $1,8 \quad(0,18)$ \\
\hline Nutrición parenteral & 9 & 15,8 & 3 & 5,8 & $2,7 \quad(0,8-9,6)$ & $2,8 \quad(0,10)$ \\
\hline Transfusión & 3 & 14,3 & 9 & 10,2 & $1,4 \quad(0,4-4,7)$ & $0,28(0,69)$ \\
\hline PICC vs CNT & 5 & 20,0 & 7 & 8,3 & $2,4 \quad(0,8-6,9)$ & $2,7 \quad(0,10)$ \\
\hline Infección local & 1 & 16,7 & 11 & 10,7 & $1,6(0,2-10,2)$ & $0,21(0,51)$ \\
\hline Colonización inserción & 3 & 13,0 & 7 & 8,6 & $1,5 \quad(0,4-5,4)$ & $0,39(0,53)$ \\
\hline Colonización conexiones & 3 & 33,3 & 7 & 8,6 & $3,9(1,2-12,3)$ & $5,0 \quad(0,03)$ \\
\hline
\end{tabular}

Tabla 3. Sensibilidad, especificidad y valores predictivos de la colonización de CVCs para el diagnóstico de ITS

\begin{tabular}{|c|c|c|c|c|c|c|c|c|c|}
\hline \multirow[t]{2}{*}{ Colonización } & \multicolumn{2}{|c|}{ SEN } & \multicolumn{2}{|c|}{ ESP } & \multicolumn{2}{|c|}{ V.P + } & \multicolumn{2}{|c|}{ V. P (-) } & \multirow[t]{2}{*}{$\mathbf{n}$} \\
\hline & $\mathbf{n}$ & $\%$ & $\mathbf{n}$ & $\%$ & $\mathbf{n}$ & $\%$ & $\mathbf{n}$ & $\%$ & \\
\hline SEG & 1 & 20,0 & 6 & 37,5 & 1 & 9,1 & 6 & 60,0 & 21 \\
\hline INS & 3 & 30,0 & 74 & 78,7 & 3 & 13,0 & 74 & 91,4 & 104 \\
\hline CON & 3 & 30,0 & 74 & 92,5 & 3 & 33,3 & 74 & 91,4 & 90 \\
\hline INS + CON vs INS o CON & 1 & 20,0 & 20 & 87,0 & 1 & 25,0 & 20 & 83,3 & 28 \\
\hline INS O CON O AM & 5 & 41,7 & 74 & 76,3 & 5 & 17,9 & 74 & 91,4 & 109 \\
\hline
\end{tabular}

SEN: sensibilidad, ESP: especificidad, INS: piel del sitio de entrada o Inserción, V.P: valor predictivo, CON: conexión, AM: INS+CON, SEG: segmento o punta del CVC.

$25,4 \%$ femorales y $20 \%$ PICC. Se observó una incidencia significativamente mayor en la colonización para las variables: ostomías (RR 2,2 IC95\% 1,1-4,3 p: 0,04) y cirugía (RR 1,99 IC95\% 2,0 1,0-3,7 p: 0,45), no se encontraron diferencias con las otras variables analizadas.

\section{Valor diagnóstico para ITS de la colonización de los CVCs}

Tanto el cultivo de segmentos de CVCs o del sitio de inserción o conexiones tuvieron una sensibilidad entre el 20 y $30 \%$, la especificidad del segmento $37,5 \%$ y mayor para los cultivos de inserción y conexión (de 78 a 92\%); los valores predictivos positivos estuvieron por debajo del 33\%. Sin embargo, al considerar colonización de inserción o conexión juntas, la sensibilidad y especificidad aumentaron a 41,7 y $76,3 \%$ respectivamente (tabla 3 ).

\section{Discusión}

En el presente estudio, la duración de los CVCs en su sitio fue de 10 a 13,9 días siendo mayor para los CNT que para los PICC, existiendo estudios que han informado en niños un promedio de 19 días ${ }^{11}$; la infusión de nutrición parenteral fue la indicación en un 50\%, y constituye siempre el uso más frecuente aún $>$ $90 \%{ }^{11}$; un $74 \%$ fueron retirados por finalización de tratamiento y un $5,9 \%$ por infección local o sospecha de ITS; otras series informan que son retirados por finalización de tratamiento $(60$ a $70,1 \%)$ y causa infecciosa $2-4 \%{ }^{11,12}$. El cambio de sistemas de infusión se observó en un rango mayor a las 72 horas recomendadas por los CDC y no hubo un protocolo de utilización de antisépticos ${ }^{1}$.

La incidencia de infección local fue de 
$5,9 \%$, la densidad de incidencia fue de $3,8 \mathrm{x}$ 1000 días/catéter (d/c) siendo ligeramente mayor en los PICC; un 66,6\% ocurrieron cuando el sitio de entrada fue en femoral; la densidad de incidencia total de ITS fue de 9 x 1000 $\mathrm{d} / \mathrm{c}$, en los PICC 24,4 x $1000 \mathrm{~d} / \mathrm{c}$ y CNT 6,2 x $1000 \mathrm{~d} / \mathrm{c}$. Se ha estimado que en los niños la frecuencia de dolor y eritema es de $13,3 \%$, edema $3,3 \%$, pus $2 \%$ e induración $1,8 \%{ }^{13}$. Se informa en hospitales de USA una incidencia de ITS asociada a PICC en neonatos de 11,6\% (DI 7,1 x $1000 \mathrm{~d} / \mathrm{c}$ ), y en CNT en miembros inferiores de 9,3\% (DI 4,8 x $1000 \mathrm{~d} / \mathrm{c})^{13}$; en unidades de pediatría, la densidad de incidencia de ITS asociada a CVCs temporales de 4,6 x $1000 \mathrm{~d} / \mathrm{c})^{14}$. En Colombia las tasas de ITS son de 11 x $1000 \mathrm{~d} / \mathrm{c}^{15}$, en neonatos 3,5\% y en pacientes pediátricos $2,4 \%{ }^{12}$.

Se encontraron como factores de riesgo relacionados con ITS, la realización de algún procedimiento quirúrgico (RR: 4,2) que el paciente estuviera ostomizado (RR: 3,9 ) y la colonización de las conexiones (RR: 3,9); los dos primeros factores se relacionaron también significativamente con la colonización del sitio de entrada y las conexiones. En Colombia se ha encontrado que la incidencia de ITS es mayor cuando hay trauma $(26,4 \%)$, patología abdominal $(14,4 \%)$ y sepsis $(10,4 \%)^{12}$. Los sitios de inserción a los cuales se atribuye mayor frecuencia de infección son los femorales y yugulares, y la menor frecuencia para los subclavios y los $\mathrm{PICC}^{1,2,14}$; los PICC, se han asociado a menor riesgo que los multilúmen ${ }^{1,2,16}$, pero un reciente ensayo clínico se demostró que presentaban tasas similares de infección que los CNT de varios lúmenes ${ }^{17}$. La infección local o la flebitis en el sitio de entrada de los CVCs usualmente no se asocia a ITS ${ }^{13}$.

Mimoz halló asociados a la colonización de los CVC la inserción en yugular (RR 2,1 IC95\% 1,24-3,24) y la utilización de povidona yodada (RR 1,87 IC95\% 1,18-2,96) para la antisepsia del sitio de inserción ${ }^{18}$.

La mayoría de las ITS fueron provocadas por Staphylococcus coagulasa (-) y cerca de una quinta parte por Cándida albicans. Estudios refieren que en neonatos y niños de todas las edades los gérmenes más frecuentes asociados a la ITS son Staphylococcus coagulasa
(-) $69 \%$, y hasta un $93 \%$ de las especies son meticillin resistentes (SMR); otros Gram $(+)$ como S. aureus y Enterococcus (9\%), bacilos Gram negativos (19\%) con predominio de Enterobacter, Pseudomonas, Klebsiella y Serratia marcescens, y Candida spp $(3 \%)^{11,13,14,17}$. También se observó predominio en la colonización de Staphylococcus coagulasa (-), se aislaron también Enterobacteriaceas, especies de Cándida, bacilos no fermentadores, S. aureus y Enterococcus. De todos los cultivos los Staphylococcus coagulasa (-) mostraron resistencia a antibióticos un $82,1 \%$ y elevada resistencia a oxacilina, aminoglucósidos y clindamicina. Entre los Gram negativos se observaron altos porcentajes de resistencia a aminoglucósidos y ampicilina sulbactam y en menor grado a cefalosporinas de primera, segunda y tercera generación. La colonización por Cándida es el mayor factor de riesgo para sepsis fúngica ${ }^{19}$; Manzoni encontró que neonatos de muy bajo peso se colonizaban en un $32 \%$ y de ellos desarrollaron infección sistémica 8\%; los factores relacionados fueron el muy bajo peso (p: 0,03 ), baja edad gestacional (p: 0,03), uso de cefalosporinas de tercera generación (p: 0,05$)$, la duración de la estancia en UCIN (p: 0,01), la colonización del CVC o del tubo endotraqueal o en más de 3 sitios (OR 4,81 IC95\% 1,8-3,9); la profilaxis con fluconazol resultó ser un factor protector independiente (p: 0,009) ${ }^{19}$.

El diagnóstico de la ITS asociada a CVC es difícil, una de las dificultades la plantea la toma de muestra para hemocultivo ya que muchas veces el difícil acceso venoso es la indicación para un CVC lo cual va en contravía con las recomendaciones internacionales para el diagnóstico ${ }^{20}$. Ante las implicaciones de retirar un CVC por la sospecha de infección y dada la patogénesis de la misma se buscó en este trabajo si la vigilancia de la colonización del sitio de entrada y las conexiones podría tener utilidad diagnóstica en ITS sin necesidad de retirar el CVC, se encontró que la sensibilidad y el valor predictivo positivo de esta técnica son muy bajas aunque la especificidad está por encima del 75\%; sin embargo, la colonización de las conexiones fue uno de las factores de riesgo identificados para ITS.

Las recomendaciones internacionales in- 
cluyen los siguientes aspectos dirigidas al control de la ITS: educación y entrenamiento a los trabajadores sanitarios, selección de CVCs y sitio de inserción, tipo (PICC, CNT y permanentes), higiene de las manos y técnica aséptica, máximas precauciones de barrera, reemplazo de apósitos y sistemas de infusión, dispositivos de seguridad para CVCs, catéteres impregnados con antisépticos/antibióticos, limpieza del sitio de inserción, profilaxis antibiótica sistémica, terapia cerrada con antibióticos y reemplazo de catéteres; dichas recomendaciones se realizan teniendo en cuenta los niveles de evidencia ${ }^{4,21}$. Recientemente se está promoviendo el uso de "Bundle" o paquetes de medidas que tienen como un mayor cumplimiento por la facilidad en la implementación, para la prevención de ITS e incluyen: higiene de las manos, máximas precauciones de barrera durante la instalación del CVC (mascarilla, gorro, guantes estériles, bata y un campo quirúrgico grande), antisepsia de la piel con clorhexidina ya que está demostrada una mayor eficacia cuando se compara con povidona yodada, selección adecuada del sitio de inserción y revisión diaria de la necesidad de conservar la línea venosa y retirarla con prontitud cuando ya no sea necesaria ${ }^{1,2,4,14,22}$.

\section{Conclusiones}

En conclusión, se encontró una tasa de infecciones similar a otros sitios con excepción de la ITS relacionada con PICC que resultó más alta; se identificaron factores de riesgo relacionados con procedimientos quirúrgicos tanto para la ITS como para la colonización; se encontró que no tienen utilidad los cultivos de vigilancia periódica de la colonización en el sitio de entrada ni las conexiones; sin embargo, se resalta la importancia de la colonización de la conexión dentro de la patogénesis de las infecciones encontradas. El perfil microbiológico predominante fue por contaminantes comunes de la piel y gérmenes hospitalarios Gram negativos con altos índices de resistencia a antibiSe recomienda la implementación del Bundle para prevención de ITS adicionando la vigilancia epidemiológica con la crea- ción de un registro que puede ser en un libro para anotaciones o en tarjetas prediseñadas donde se puedan vigilar los aspectos más importantes dentro del seguimiento de los CVCs; así mismo enfatizar en la uniformidad de criterios para el cambio de sistemas de infusión y la utilización de antisépticos. Es necesario realizar un monitoreo del comportamiento de los perfiles de sensibilidad y resistencia de los gérmenes locales a los antibióticos.

\section{Referencias}

1.- O’Grady NP, Alexander M, Dellinger EP, et al: Guidelines for the prevention of intravascular catheter-related infections. Centers for Disease Control and Prevention. MMWR Morb Mortal Wkly Rep 2002; 51: 1-29.

2.- Trautner B, Darouiche RO: Catheter-Associated Infections: Pathogenesis Affects Prevention. Arch Intern Med 2004; 164: 842-50.

3.- Darouiche RO: Prevention of infections associated with vascular catheters. The Int J Artif Organs 2008; 31(9): 810-819

4.- O'Grady NP, Alexander M, Burns LA, et al: Guidelines for the Prevention of Intravascular Catheter-Related Infections, 2011. Centers for Disease Control and Prevention (CDC). http://www.cdc.gov/hicpac/BSI/ BSI-guidelines-2011.html, última visita: 20-9-2011.

5.- Horan TC, Andrus M, Dudeck MA: CDC/NHSN surveillance definition of health care-associated infection and criteria for specific types of infections in the acute care setting. Am J Infect Control 2008; 36 (5): 309-32.

6.- Flynn PM, Barrett FF: Infecciones asociadas a los dispositivos médicos. En: Behrman, Kliegman, Jenson. Ed. Tratado de Pediatría de Nelson, España Elsevier, 2004; 858-61.

7.- Donlan RM: Biofilms: Microbial Life on Surfaces. Emerging Infectious Diseases 2002; 8 (9): 881-90.

8.- Donlan RM: Biofilms and Device-Associated Infections. Emerging Infectious Diseases 2001; 7 (2): 277-81.

9.- Maki DG, Weise CE, Sarafin HW: A semiquantitative culture method for identifying intravenous-catheterrelated infection. N Engl J Med 1977; 296 (23): 1305-9.

10.- Bouza E, Liñares J, Pascual A: Diagnóstico microbiológico de las infecciones asociadas a catéteres intravasculares 2004. http://www.seimc.org/documentos/ protocolos/microbiologia/, última visita: 9-9-2011.

11.- Pawinska A, Grochowska M, Piedgon G, Dzierzanowska D: Monitoring Infection Complications Associated with Intravascular Therapy in the Children's Memorial 
Health Institute. Indoor Built Environ 2006; 15 (1): 2934. http://ibe.sagepub.com/cgi/reprint/15/1/29, última visita: 9-9-2010.

12.- Echeverri S: La Cateterización Venosa Central en la Fundación Santa Fe de Bogotá. http://www.tensiometrovirtual.com/upload/EF-001_g.pdf, última visita: 19-9-2011.

13.- Hoang V, Sills J, Chandler M, Busalani E, Clifton R, Modanlou HD: Percutaneously Inserted Central Catheter for Total Parenteral Nutrition in Neonates: Complications Rates Related to Upper Versus Lower Extremity Insertion. Pediatrics 2008; 121 (5): e1152-9. http:// pediatrics.aappublications.org/cgi/content/full/121/5/ e1152, última visita: 6-6-2010.

14.- Edwards JR, Peterson KD, Mu Y, et al: National Healthcare Safety Network (NHSN) report: Data summary for 2006 through 2008, issued December 2009. Am J Infect Control 2009; 37: 783-805.

15.- Álvarez CA, Cortés JA, Gómez CH, Fernández JA, Sossa MP, Beltrán F: Guías de práctica clínica para la prevención de infecciones intrahospitalarias asociadas al uso de dispositivos médicos. Infectio 2010; 14 (4): 292-308.

16.- Zurcher M, Tramér MR, Walder B: Colonization and Bloodstream Infection with Single-Versus Multi-Lumen Central Venous Catheters: A Quantitative Systematic Review. Anesth Analg 2004; 99: 177-82.

17.- Safdar N, Maki DG: Risk of Catheter-Related Bloodstream Infection with Peripherally Inserted Central
Venous Catheters Used in Hospitalized Patients. Chest 2005; 128: 489-95. http://chestjournal.chestpubs.org/ content/128/2/489.full.pdf+html, última visita: 06-062010.

18.- Mimoz O, Villeminey S, Ragot S, et al: ChlorhexidineBased Antiseptic Solution vs Alcohol-Based PovidoneIodine for Central Venous Catheter Care. Arch Intern Med, 2007; 167 (19): 2066-72. http://archinte.ama-assn. org, última visita 17-1-2011.

19.- Manzoni P, Farina D, Leonessa ML, et al: Risk Factors for Progression to Invasive Fungal Infection in Preterm Neonates With Fungal Colonization. Pediatrics, 2006; 118 (6): 2359-64 http://pediatrics.aappublications.org/ content/118/6/2359.full.pdf + html, última visita 17-12011.

20.- Falagas ME, Kazantzi MS, Bliziotis LA: Comparison of utility of blood cultures from intravascular catheters and peripheral veins: a systematic review and decision analysis. J Med Microbiol. 2008; 57: 1-8. http://jmm. sgmjournals.org/cgi/reprint/57/1/1, última visita: 3-122010.

21.- Yokoe DS, Mermel LA, Anderson DJ, et al: A Compendium of Strategies to Prevent Healthcare-Associated Infections in Acute Care Hospitals. Infec. Control Hosp Epidemiol 2008; 29 (S1): S12-S21.

22.- Institute for Healthcare improvement. Implement the Central Lines Bundle. http://app.ihi.org/imap/tool/\# Process $=e 876565 d-f d 43-42 c e-8340-8643 b 7 e 675 c 7$, última visita: $15-9-2011$. 